immunity and antibody-dependent enhancement// Kyoto University, Kibi International University Cambridge University - May 02, 2020 Version 1 DOI: 10.33774/coe-2020-fsnb3 [Electronic resource] URL: https://www.cambridge.org/engage/coe/articledetails/5 ead2b518d7bf7001951c5a5

95. Physicists received a previously unseen type of matter from light/ / C 2020 Online edition «Вести.Ру» - 26.09.2013 [Electronic resource] URL: https://www.vesti.ru/doc.html?id=1134707\& cid=2161

96. Brian Swingle. Spacetime from Entanglement/Annual Review of Condensed Matter Physics. Volume 9, 2018-pp. 345-358 [Electronic resource] URL: https://doi.org/10.1146/annurevconmatphys-033117-054219

97. Christopher R. Moon, Laila S. Mattos, Brian K. Foster, Gabriel Zeltzer \& Hari C. Manoharan Quantum holographic encoding in a two-dimensional electron gas/Nature Nanotechnology. Volume 4, 2009 - p.167-172/ [Electronic resource] URL: https://www.nature.com/articles/nnano.2008.415
98. Poincaré H. La théorie de Lorentz et le principe de réaction (French) / / Archives néerlandaises des sciences exactes et naturelles - 1900. - Vol. 5. - P. 252-278

99. Perelman, Grisha (November 11, 2002), the entropy formula for the Ricci flow and its geometric applications, arXiv:math.DG/0211159 [math. DG]

100. Perelman, Grisha (March 10, 2003), Ricci flow with surgery on three-manifolds, arXiv:math.DG/0303109 [math. DG]

101. Perelman, Grisha (July 17, 2003), Final extinction time for the solutions to the Ricci flow on certain three-manifolds, arXiv:math.DG/0307245 [math.DG]

102.

Bo-Sture K. Skagerstam, Karl-Erik Eriksson and Per K. Rekdal Causality in quantum field theory with classical sources // Journal of Physics $\begin{array}{lllll}\text { Communications. } & 3 & \text { (2019) } & 8 & 082001\end{array}$ DOI: 10.1088/2399-6528/ab3c1c [Electronic resource] URL: https://inspirehep.net/literature/1651252

\title{
PHYSICAL FOUNDATIONS OF UNIFIED KNOWLEDGE
}

\author{
Romashov1 A.N., \\ Tsygankov2 S.S., *, \\ Tsygankov Jr. S.S. 3 \\ ${ }^{1}$ PhD, Leading researcher, Russian Academy of Sciences, \\ Moscow, Russia \\ ${ }^{2}$ PhD, corresponding member, Russian Academy of Natural Sciences, \\ Moscow, Russia \\ ${ }^{3}$ PhD, vice president, Association for the Protection of Population, \\ Moscow, Russia \\ * ORCID 0000-0002-8179-672X \\ DOI: $10.31618 /$ asj.2707-9864.2020.2.41.30
}

\begin{abstract}
A physical model of the Universe is proposed, which is based on the idea of the world as two interconnected branches of a single evolutionary process. One branch is the creation of a postulated primary particles - forms and their cells of a multilevel oscillating structure - a Unified set of forms (USF), which, in the model, is space; second, the formation of material realities by combining the same forms in a structured space. The interaction of the same particles representing "matter" and "space" gives a strict coordination of their development at all scale levels, from elementary particles, atoms, up to Homo sapiens.

USF is the "constructor" of all material realities, and is responsible for their interactions - the forces that arise between them. So, gravitational forces are the stresses in the USF caused by its deformation ("space curvature") by material objects. This explains its long-range and high-speed performance, which was clearly shown by experiments on elastic models.

USF also gives wave properties to all particles moving along its corridors, which determine the transmission properties of space. At this stage of knowledge, USF provides a clear physical explanation for the de Broglie hypothesis, the Heisenberg uncertainty principle, and some other physical phenomena. The constant interaction and mutual development of the USF and the material world give the direction of the evolution process of the Universe.
\end{abstract}

Keywords: particles - forms, spin, the Unified Set of Forms (USF), structuring of space; hexagonal oscillation cells; structuring of matter, corridors of USF, wave-particle duality

"We come to a strange conclusion: now it begins to seem to us that space plays a primary role; matter must be obtained from space, so to speak, at the next stage" [1].

\section{Introduction.}

There is currently no comprehensive model covering the entire universe: particles, interactions and space, dark matter and energy. The Standard Model, Special relativity (SR) and General relativity (GR), strings theory, preons, E8, structural vacuum, and others do not give a unified picture of the material world. It is probably impossible to describe the world in parts. None of the models for the development of the structures of the material world will be successful if it does not take into account the existence of a space with physical properties. Therefore, the construction of quantum mechanics is becoming more complex and has long lost any physical interpretation. On the other hand, for several centuries of the existence of modern science, it is intuitively felt that the Universe is a unified 
mechanism that can be described in a clear and understandable deterministic form [2-4]. In this work, such an attempt was made to build a comprehensive model of the Universe, within the framework of Unified knowledge, in which matter and space are two basics of a unified physical process [5].

\section{Postulates.}

Like any other physical model, Unified knowledge is based on certain postulates that will have to be taken on faith.
The first postulate: Matter has a limit of fragmentation, i.e. all material objects, bodies and their systems are based on some simple particles protoforms, meaning that over time all objects of matter and space will arise from them.

The second postulate: the initial protoforms have the property of mutual attraction through empty gaps between them. Following the laws of dialectics, along with the introduced discreteness - the first opposite, the second postulate introduces continuity - the second opposite.

\section{$\mathrm{S}=1 / 2 \mathrm{~h}$ образ}

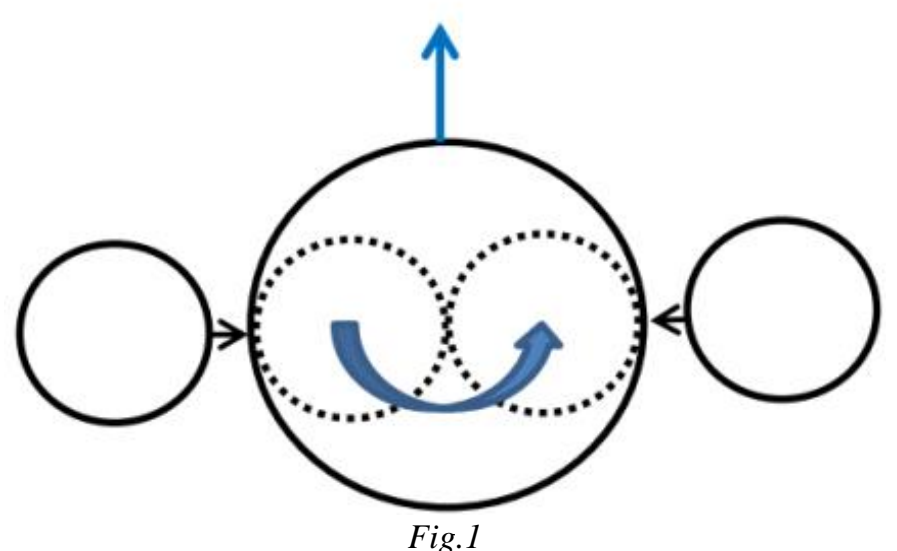

The third postulate: two protoforms, that have reached the point of meeting due to mutual attraction, capture each other, unite into a single particle that receives a rotational movement - this is the act of "negation-negation" of both opposites: the first two postulates. In this case, the first real particle is born and its fundamental property - movement appears. The particle is called the form (forming the world). The form has acquired a single moment of rotational motion (spin) (Fig. 1).

\section{Interaction of forms.}

Thanks to spin forms become qualitatively distinguishable from each other, both in the orientation of the axes of rotation, and in two possible directions clockwise and counterclockwise. If the original types were all the same and any pair of them could be combined with each other, then the combination of forms should already become selective. If we choose the form with a certain spin orientation, then only the form with a parallel spin can be combined with it, so that their rotations on the contact are compatible.

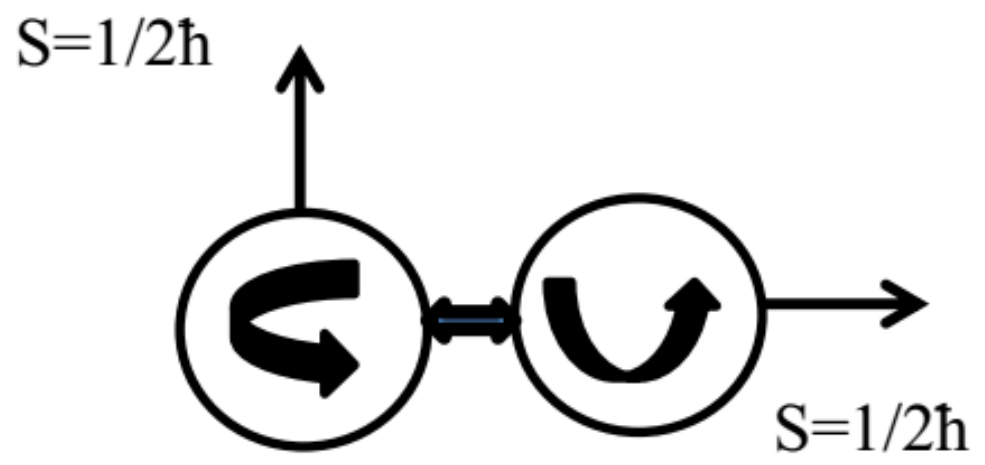

Fig. 2

Two forms whose spins are mutually perpendicular are not capable to combining. The force of attraction brings them into contact, and the incompatibility on contact throws them away. At the same time, they will make oscillatory movements (Fig. 2).

\section{Two branches of world development.}

Hence follow two possible branches of the development of the entire initial substance. Forms that are not capable of mutual association will be located in it according to the principle of mutual perpendicular of the spins of neighboring forms. They will give the entire space structure and will serve as a kind of frame for it. Being attracted and bouncing from each other, 
they are in constant oscillatory motion. Let's call this part of the initial substance a Unified Set of Forms (USF), since it will represent an absolutely stable combination of these forms.

The second branch of our Universe is represented by forms that can be combined and have the compatibility of movements on contact, i.e. parallel spins. This branch will continue the process of development of the initial substance: forms will combine in pairs, triples, and so on, creating first elementary particles, then atoms and molecules, and finally living organisms, including Humans.

\section{Structuring the space (USF).}

\begin{tabular}{|c|c|c|c|c|}
\hline$-0>$ & $\odot$ & $\phi$ & + & $\theta$ \\
\hline$\oplus$ & $\stackrel{4}{\varphi}$ & $-\infty$ & $\odot$ & ధ \\
\hline$\phi$ & +0 & $\oplus$ & $\phi$ & $-\infty$ \\
\hline-0 & $\odot$ & ф́ & +0 & $\oplus$ \\
\hline$\oplus$ & $\phi$ & $-\infty$ & $\odot$ & ф) \\
\hline 8 & $+a$ & $\oplus$ & $\oint$ & $-\infty$ \\
\hline
\end{tabular}

Fig.3

Let's consider structuring by the first type. Let's imagine that at some initial moment of time the Unified Set of Forms construct a stationary medium of particles with perpendicular spins at neighboring points (Fig. 3). The forces of attraction between the particles balance each other in pairs, and the entire system is in a state of unstable equilibrium.

The simplest model of such unstable equilibrium was reproduced experimentally in the one-dimensional case. Cylindrical magnets were located along their axes in a line at a certain distance from each other (Fig.4).

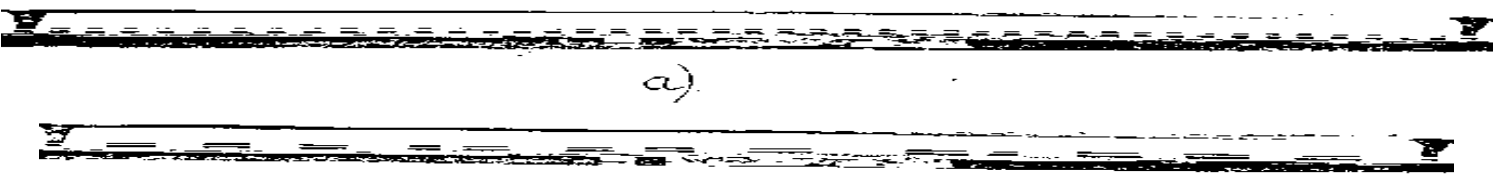

5).

Fig. 4 


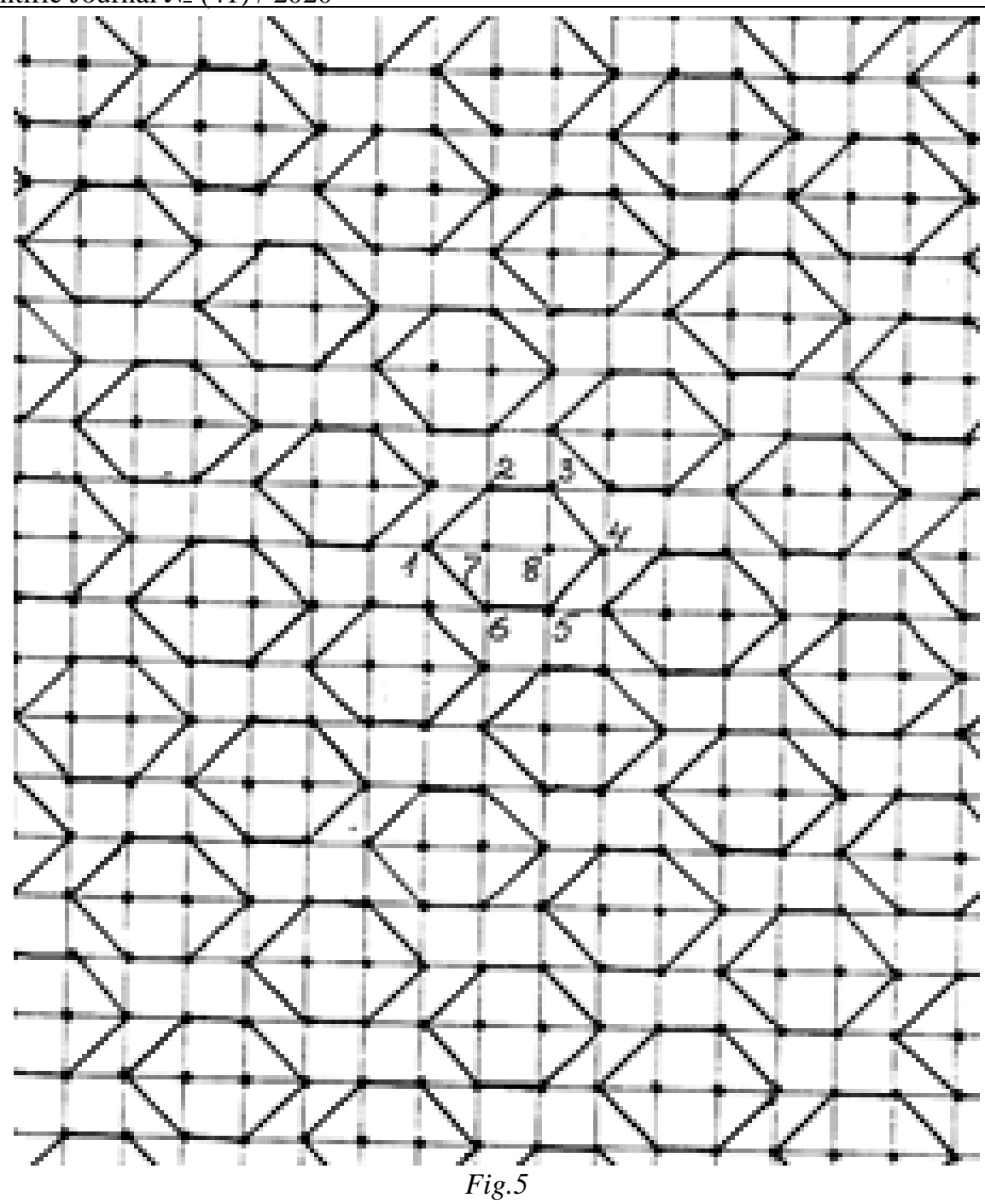

At the slightest displacement of any of the magnets the total equilibrium is disturbed - they all come into motion and as a result, cells of these magnets, form an avalanche, each of which is 2-4 (average -3) magnet (4b). Although each of these groups is influenced by the neighboring ones, the force of attraction contains is already less than the force of friction. Therefore, the new state is stable. But theoretically, you can imagine the friction force equal to zero. In this case, the resulting groups of magnets will in turn begin to combine into larger groups, those into even larger ones, and so on, until all the magnets are combined into a single discrete "rod".

Now let's consider the interaction of forms in the plane. It is enough for one of them to shift to one side or the other in the direction of the neighboring form, as the general balance will be disturbed. First, the forms will be combined in pairs, then in triples and so on. A qualitatively obtained, hexagonal version of such a new structure (cells) of eight images, capable of structuring the entire USF, is shown in Figure 5.
At the same time, the new system of forms will be more stable - along with interacting forms in individual cells, their groups (cells) will also interact in it. They, like single forms, are attracted to each other and bouncing, will oscillate with a lower frequency.

The next, larger version of structuring the forms system by joining in a group takes a three-level system, in which, along with the free forms of the first level, working mainly inside the first hexahedral cells of the second level, a new hexahedral cell of the third level appeared, which united the first seven hexahedrons (elements of the second level).

The process of structuring the system of forms on the plane can be continued: seven structural elements of the third level are able to combine into a new element of the fourth level (Fig. 6) and so on. Thus, in USF we have an arbitrarily large (but finite) set of structures that are hierarchical in nature and obey a strict ratio in size and number of structural elements of different scales. Each level of association will have its own frequency of oscillations of the forms - cells. 


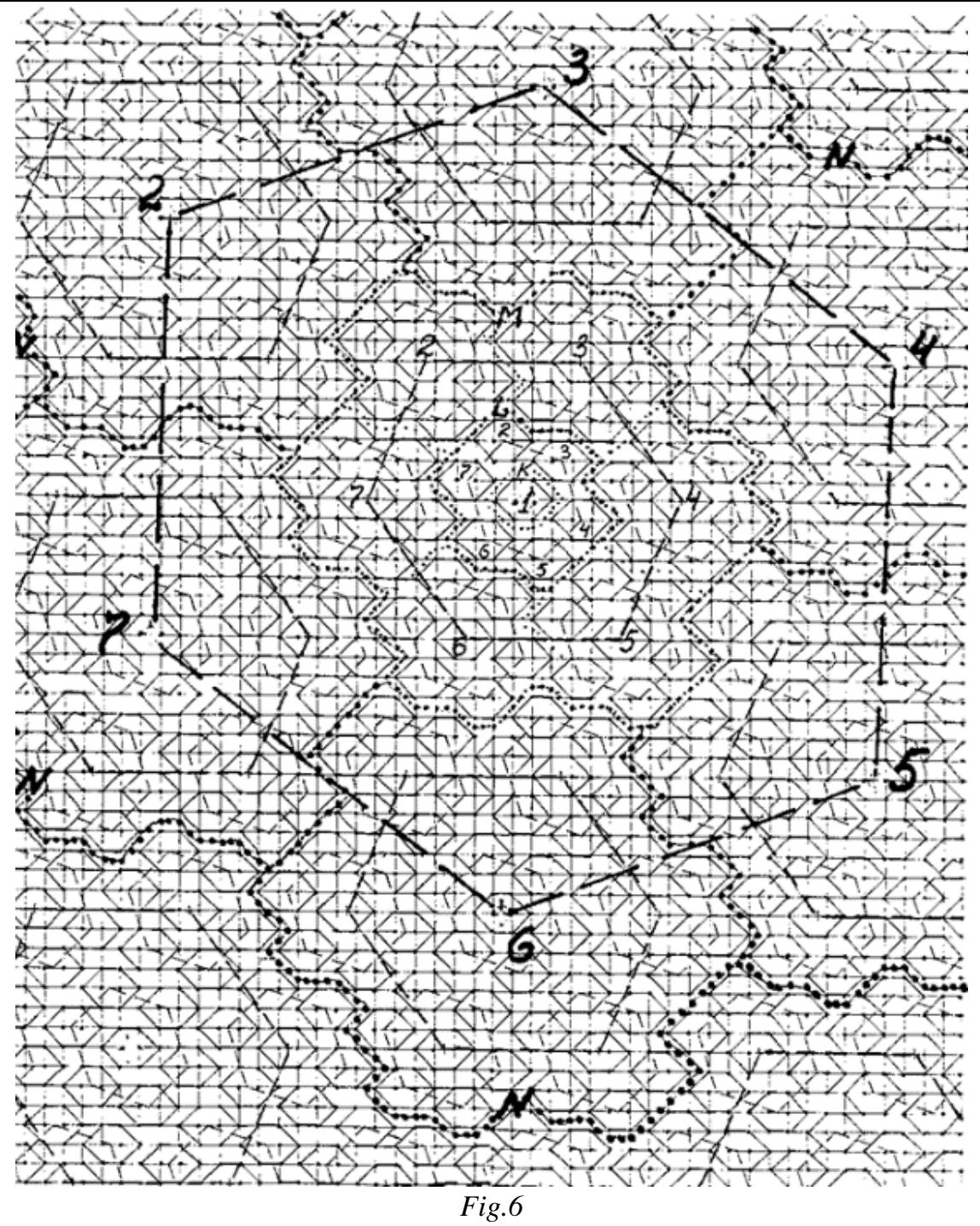

And if we now take any material system, whether it is a galaxy, a Solar system, the Earth, or some fragment of it, then in each of these systems there will be a whole set of structural elements in the USF, starting with the size of this material system and ending with the micro level. Oscillations of these structures will determine the entire process of formation and development of this material system, as a whole, and all its components.

Three-dimensional structuring of USF, while intuitively, can be assumed to follow two types: in planes, and simultaneously in a perpendicular direction, when the structure of some "first" plane will be exactly reproduced in all parallel planes. They will form hexagonal "prisms" that pulsate along these axes, along with radial pulsation.

In the future, the first prisms will begin to combine with each other, like triples of real magnets without friction (Fig.4). This will be followed by the third level of integration, the fourth, and so on to the "border" of the environment.

\section{Structuring of matter.}

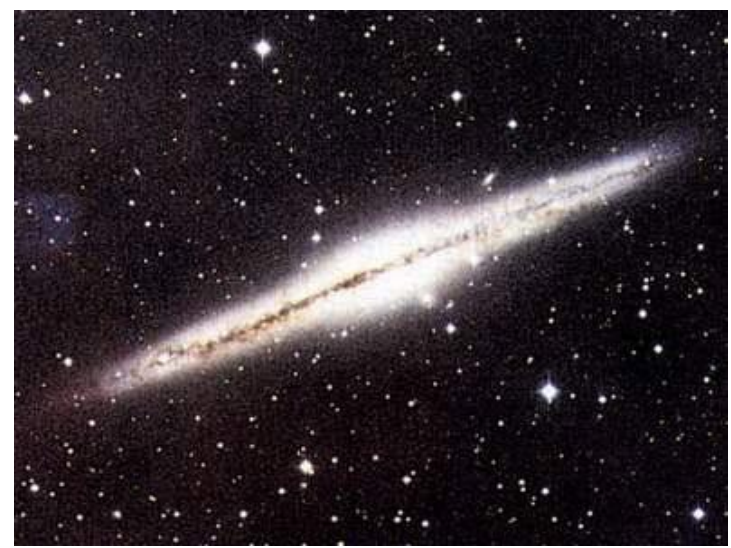

Fig.7 
If we now "throw" forms of the material world (with parallel spins) into such a pulsating medium (USF), then their general behavior will be determined by the processes of the micro level, at which the combining of individual forms into elementary particles, atoms, etc begins, and the process of contraction of all material clumps to a certain central plane, and in the plane to the center of the primary cell.
From here, obviously, one of the main features of the structure of our Universe at the macro level follows - all cosmic bodies are combined into star-planet and galactic systems that have a clearly expressed flat shape (Fig.7). USF, both as a whole and within all of its cells, is characterized by a general regularity: the average bulk density of images increases towards the centers of the cells and their axial planes.

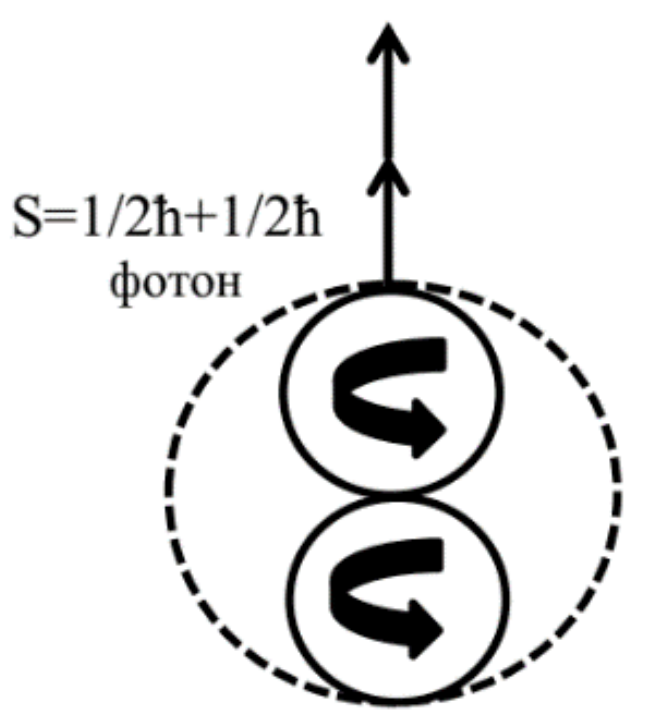

Fig.8

The formation of particles of matter. At the micro level, the formation of material particles occurs due to the coalescence of forms with parallel spins.

Photon.
If the spins are directed in parallel, then they can connect only in series so that their rotations coincide. So, presumably, a photon is created from two forms, with a total spin of 1 (Fig.8).

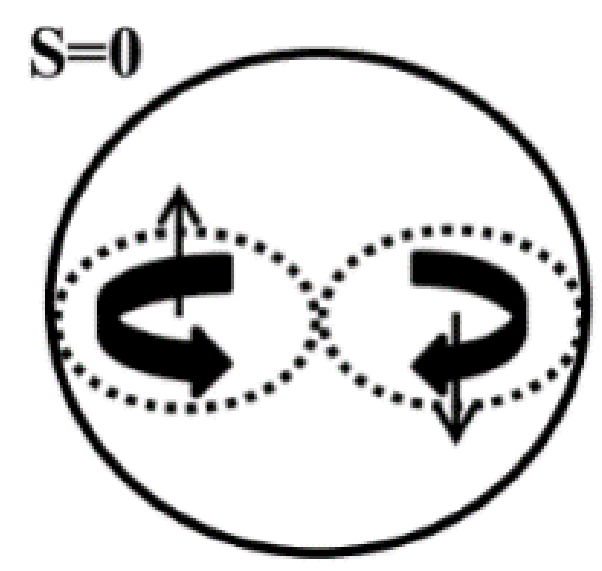

Fig.9

\section{Mass of particles.}

Forms with antiparallel spins cannot connect in series, due to incompatibility of movement, but can combine in the plane of rotation. In this case, a particle having a mass with zero spin is created (Fig.9). Call it em (mass element).

It seems that of them, the further development of matter in two directions is possible. One is the enlargement of mass, and the other is the creation of particles with a new quality.

By the type of mass enlargement, the figure 10 shows the sequential association of particles into larger structures, analogues of strings with a total zero spin. From them in the future will be collected masses of all particles. The possibility of the decay of "matter" into energy - "photons" circled in blue is shown. 


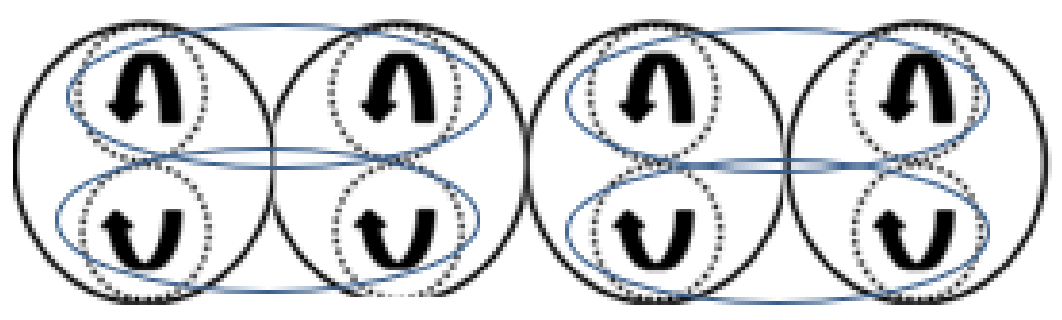

Fig.10.

\section{Lepton number.}

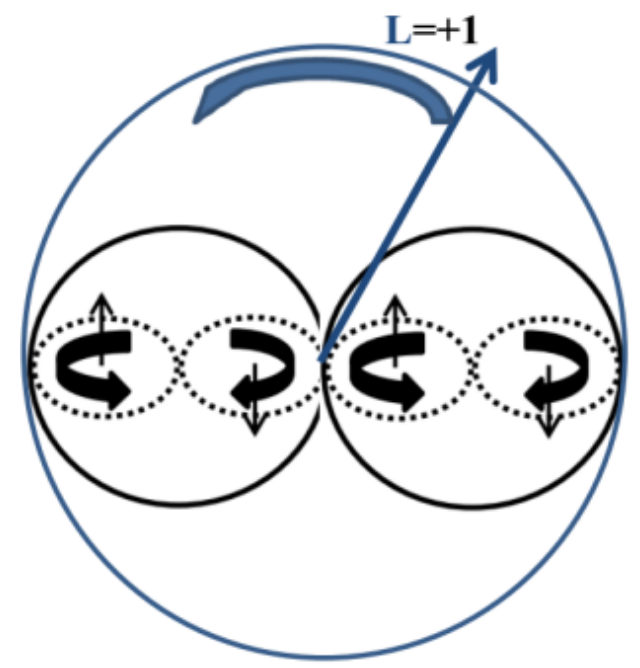

Fig.11

The figure 11 shows the union of two particles of two forms each in the plane of their rotation. As you can see, the rotation of the particles on the contact are compatible. As follows from the third postulate, two attracting particles by mutual capture form a new rotational pair with an axis of rotation directed orthogonally to the plane of the figure (with a rotation of 90 degrees). The appearance of a new rotational particle is the birth of a new material quality, it receives a new characteristic. An analog in quantum mechanics, apparently, is the lepton number.

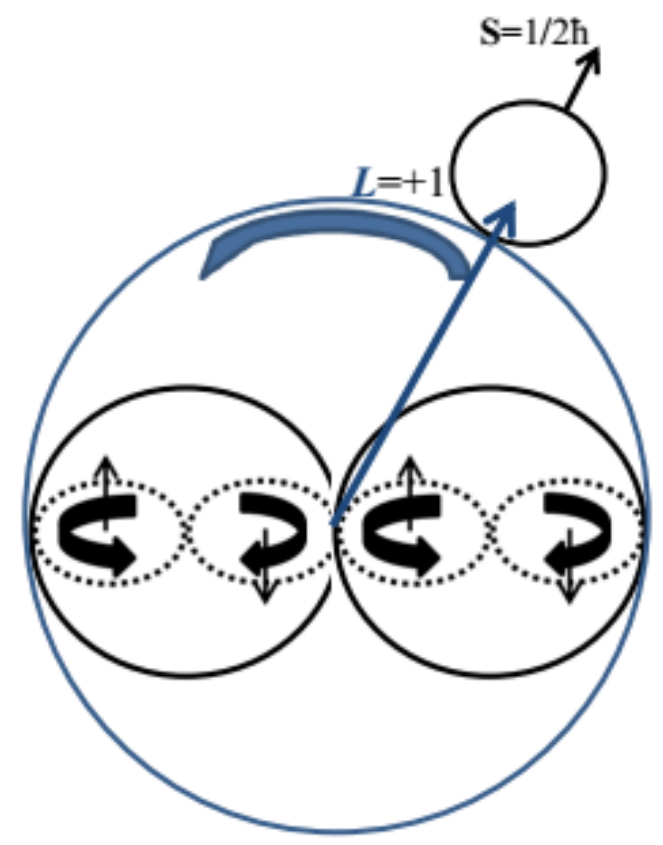

Fig.12 
Neutrino. Further development can go by joining a free form to a new particle with a spin direction compatible with its new torque (Fig.12). They can be combined according to the principle of series connection (like forms in a photon). As a result, a particle appears, consisting of five images and characterized by two quantum characteristics: a spin equal to $1 / 2$ (due to the image), and a lepton number (due to a new rotating pair of particles). The real analogue of such triples, apparently, is the first lepton the electron neutrino.

Neutrinos of the second and third generations can be formed in the future according to this principle due to the addition of the corresponding masses.

\section{Electric charge.}

It can be assumed that, according to the same scheme, two formed particles from four forms will combine and a new particle will be created with a new quality - electric charge (e/3). The combination of these three particles, the addition of a particle characterizing a lepton number and a free form to them, will create a new particle that will already be characterized by three quantum numbers (spin is $1 / 2$, lepton number and electric charge). An analogue of such a particle can be an electron.

Most likely, it is necessary that a chargeless particle - "mass" - be attached to this particle. The number of these attached particles (their mass) will determine the generation of particles with an electric charge of -1 : the first generation is an electron, the second (muon) and the third (tau).

To preserve the moment of momentum, the formation of charge particles should occur in pairs. In the course of development, one of these paired particles can already acquire independent existence (electrons) at this level, while the opposite particles (quasipositron) continue the process of further enlargement (combining with their own kind) and, as it were, form the basis for particles of the following scale levels, for example, quarks with charges that are multiples of $1 / 3$.
Quarks. Protons. Neutrons. On the way to the complication of matter, the next quality is the baryon number. The process of formation of particles with this charge is likely to repeat the process of formation of lepton and electric charges due to the formation of new paired particles with a new rotation of different signs, due to the combination of particles of the previous level.

The subsequent attachment of quasipositron clumps to them, obviously, led to the formation of quarks and their further unification already on the scale of some "nucleon" cells.

Particle antiparticle.

In the formation of new particles due to the combination and joint rotation of particles of the previous level, to preserve the moment of momentum, they were born in pairs: an antiparticle particle with rotation both clockwise and counterclockwise. All particles with different quantum numbers: lepton, baryon, electric and color, have antiparticles.

There are no antiparticles in the form and a pair of forms connected, both in series - a photon, and antiparallel, forming a mass. Therefore, the Universe has particles and antiparticles, but there is no antimatter, because there is no "anti-mass".

Atom. USF structures are also of fundamental importance in the assembly of atoms. The force of attraction inherent in nuclei attracts electrons to them, but they cannot "contact" with each other - they are not allowed to the nuclei surrounding their forms of the primary cell of the USF. The gaps between the primary cells ("corridors" of the USF) obviously make up the possible location of electrons. Its existence can be figuratively imagined as a constant passage through an annular system, on the sides of which oscillating forms of neighboring cells are located, giving electrons rotation in orbit. The principle of Pauli becomes clear. Two particles with the same spin in the same orbit, in the same corridor, are incompatible in their motion.

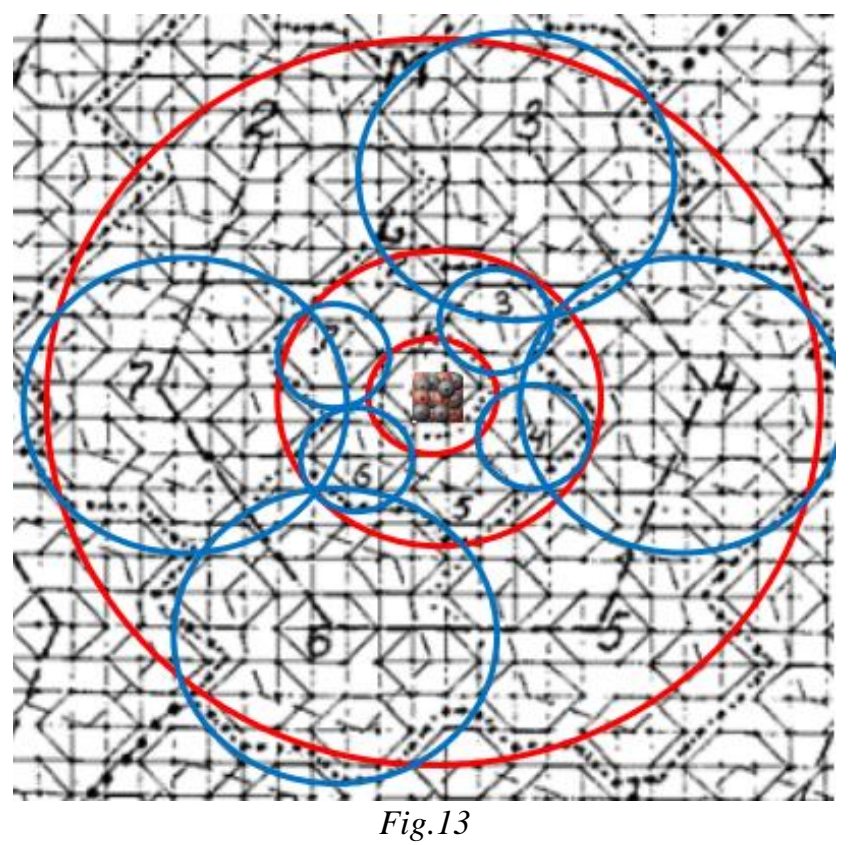


The filling of the orbits with electrons will be determined by the capabilities of the free corridors between the USF cells. After filling the closest corridor - the orbits near the nucleus (level K), new electrons tending to the nucleus will fill the corridors of the second level (L) (Fig.13). First, a circular corridor between cells of the next level around the nucleus (sorbital). Between the $\mathrm{K}$ and $\mathrm{L}$ levels there are places between cells that can be filled with electrons to form three dumbbell-shaped $\mathrm{p}$ - orbitals, etc.

At the f-orbitals and the Q-energy level, the atomic stage of the complication of matter ends. The size of the nuclei reaches critical values at which the USF structures become impassable for them. The phenomenon of radioactivity is apparently associated with this.

\section{Structures USF. \\ Interactions.}

The single basis of all the forces between the particles is the initial force of attraction between the forms. As forms combine with the creation of new particles and their cells, new total forces arise between the particles and their cells. Such a first force, most likely, was the weak interaction inherent in all particles: leptons and quarks.

Particles and their cells with a new quality - an electric charge, created a new interaction electromagnetic. The limited speed of its transmission indicates that its particles, photons, carry it.

A strong interaction appears between the USF cells, which have collected quarks in the material half, and then nucleons.

\section{Gravity.}

In the process of the formation of matter, both the unification of its constituent forms and the contraction of the USF forms with which they are connected by the initial force take place.
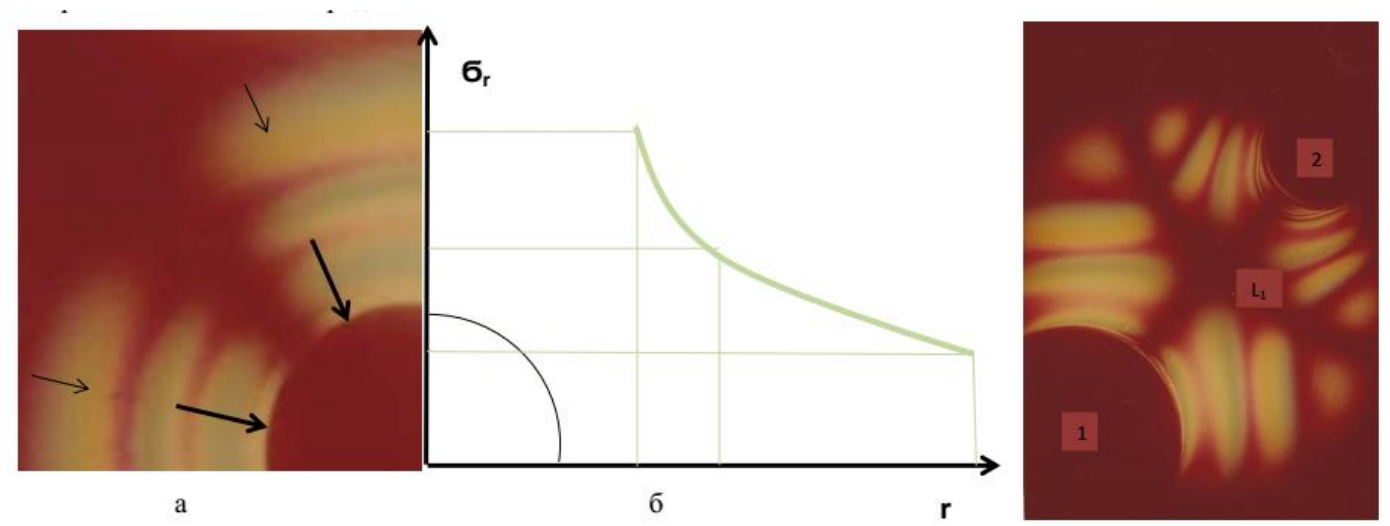

Fig. 14

a) A photograph of a pattern of colored stripes (isochrom) in a single-core model. The arrows indicate the direction of the stress $\sigma r ; b)$ the distribution of $\sigma r$ in relative units (serial number of the green bar);

c) the interaction of two bodies.

This increases the density of USF forms in the body, causes an additional force, the force of gravity, towards it. In this case, the field of deformations of the USF around the body, the so-called "curvature of space", will be its gravitational field. This is well visualized by experiments simulating elastic stresses in core models, where $6 r \sim r^{-2}$ (fig. 14b).

Long range gravity. Signal transmission rate through USF.

Thanks to USF, all particles and objects are interconnected through its structure. The local perturbations of the USF that they cause almost instantly propagate to the farthest distances.
This is embodied in quantum entanglement when the quantum states of two or more objects are interdependent. An experimental verification of the speed of such a signal is at least hundreds of thousands of times faster than the speed of light.

Structures USF in nature. The degree of manifestation of USF structures in natural processes depends on the degree of equilibrium of the transition from one state to another. The photo shows basalt hexagonal columns on the Giant's dam on the coast of Northern Ireland, formed during crystallization during the slow cooling of volcanic lava (Fig. 15). 


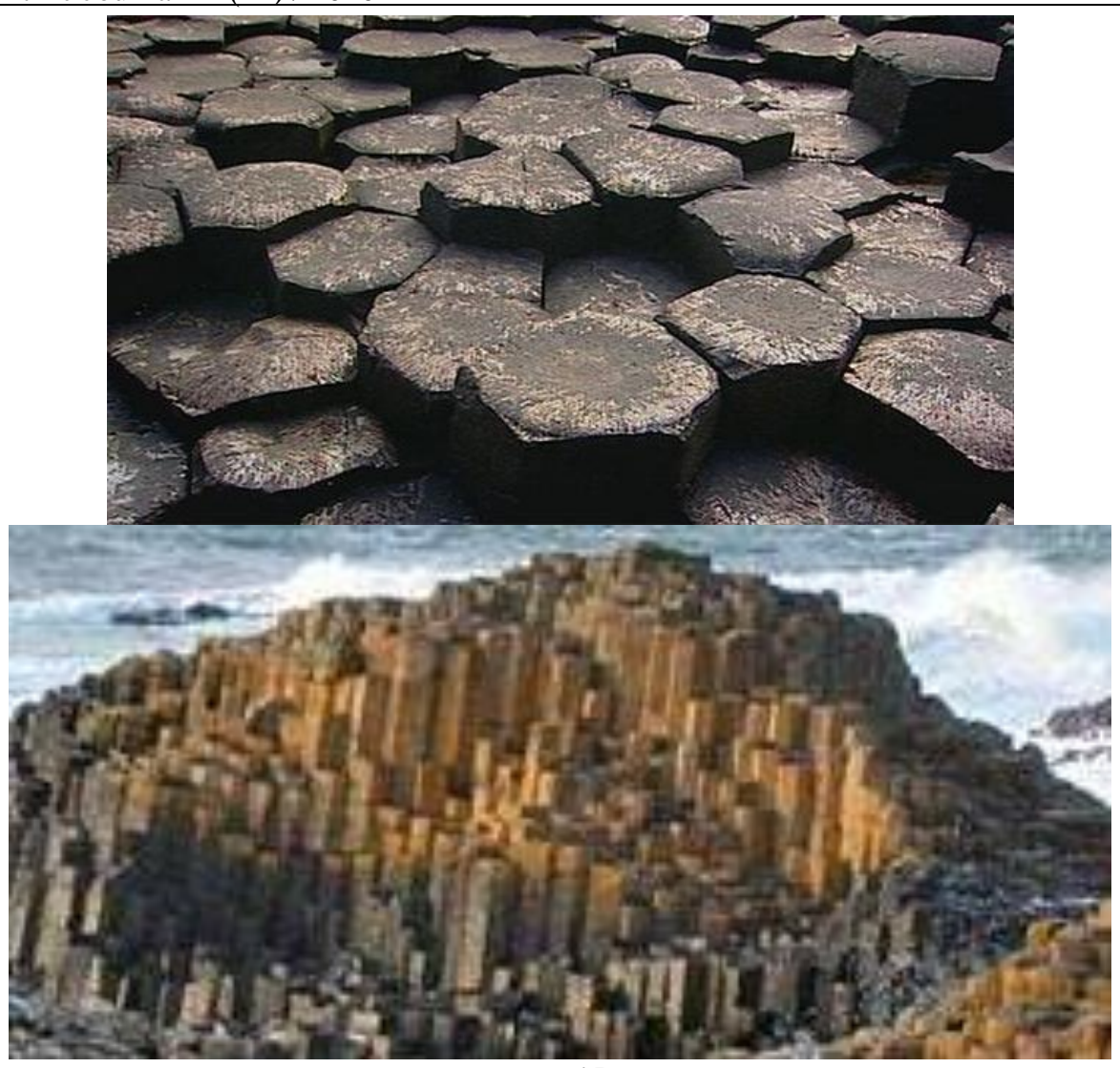

Fig.15

A similar picture occurs during the compaction of sedimentary rocks or the drying of wet clay soils (Fig.16). The biaxial tension that occurs in the above examples in the upper layer of a crystallized or shrinking volume can be realized by any network of cracks. But the one that the USF imposes with its additional field is implemented.
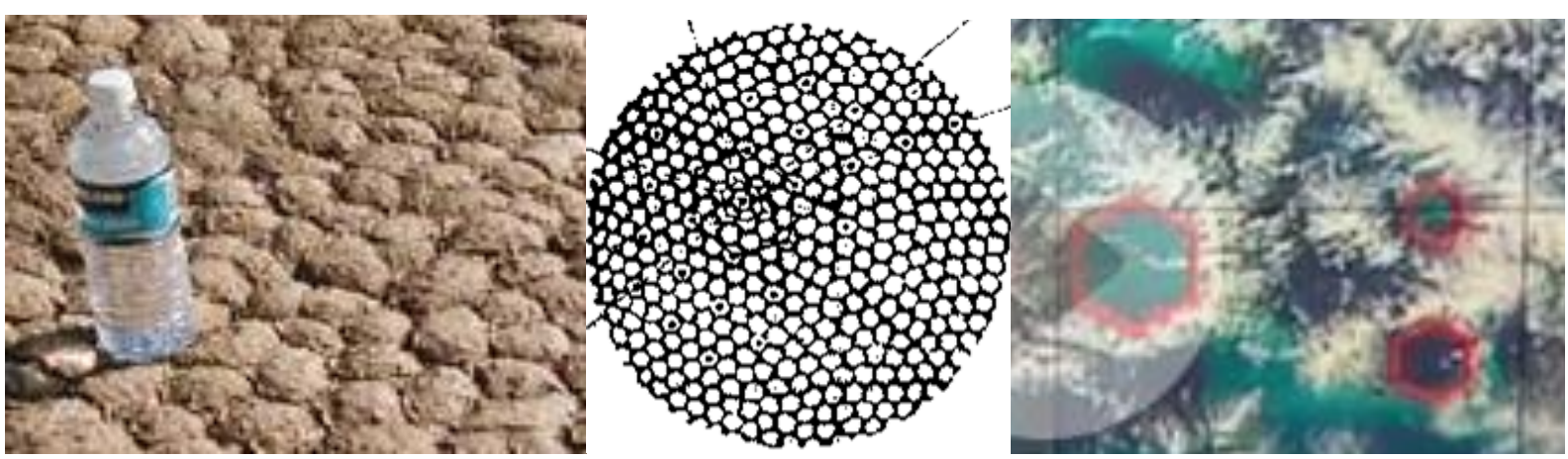

Fig. 16. Formation the system of cracks during drying of wet soils,

Benard cells in convective liquid, cells in clouds over the Atlantic. 


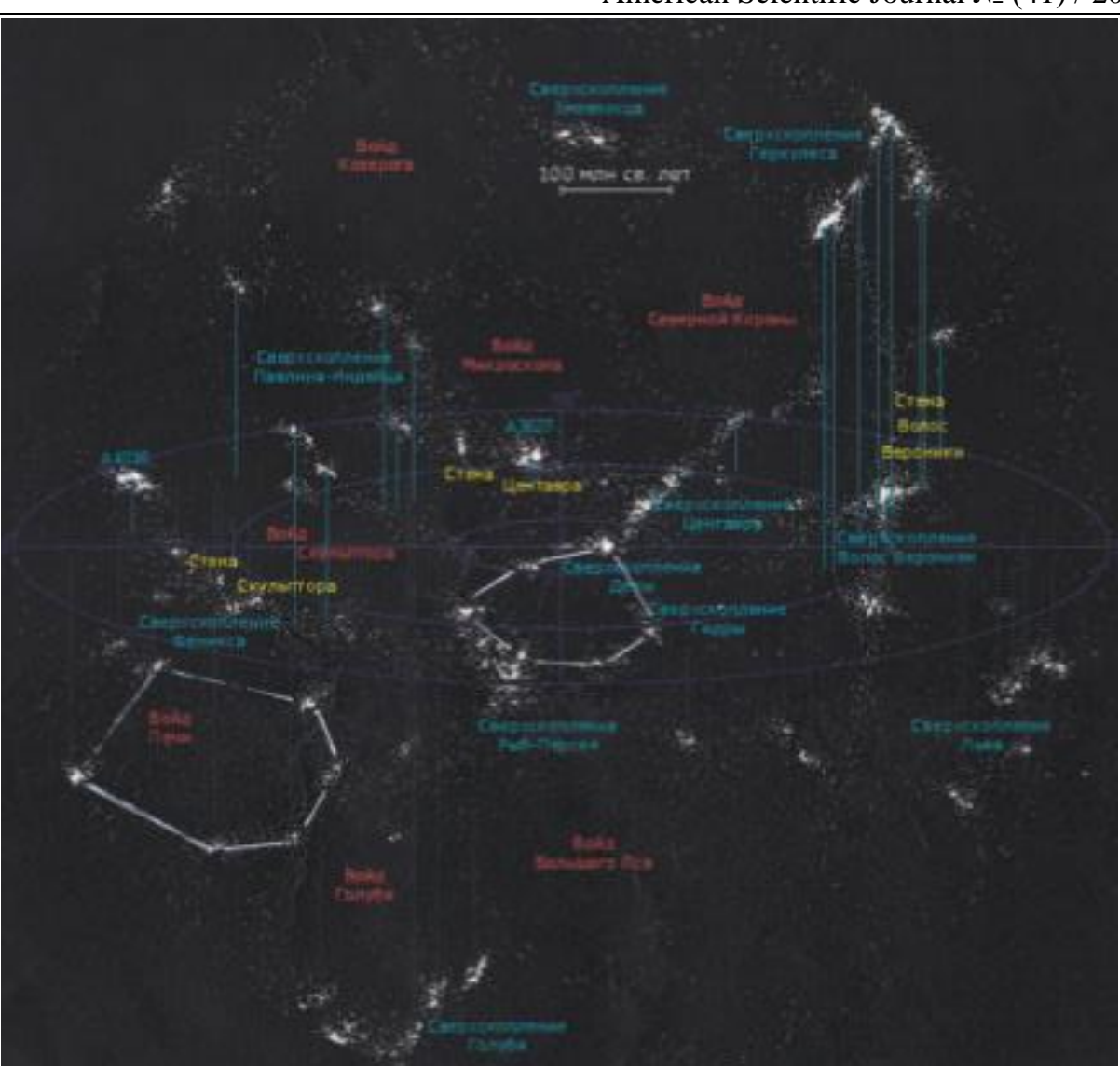

Fig.17

The hexagonal shape of the "honeycombs" is USF well known to astrophysicists studying large-scale structures of the Universe (Fig.17). The vibrational movements of structures USF of the galactic level determine the process of formation and development of galaxies and their clusters, collecting them in sparseness corridors of USF, thread-like walls of hexagons separated by voids, in which there is practically no luminous matter.

It is the strict dynamic orderliness of NATURE that exists in the "non-material" world that is clearly manifested in the uniformity of natural (material) formations.

\section{Modern physics and USF.}

Modern physics does not take into account the existence of the ether and the USF as well. Its equations were initially selected in such a way that they satisfy the requirements of the real existence and behavior of material systems. There is no physical explanation for the adequacy of many such models and reality.

Perhaps this is impossible to explain, because it turned out to be lost the main principle of the world USF, which actually acts as the Creator of all the realities of the material world. It provides a simple and convincing explanation of many physical models and mathematical equations in modern science.

\section{Wave-particle duality.}

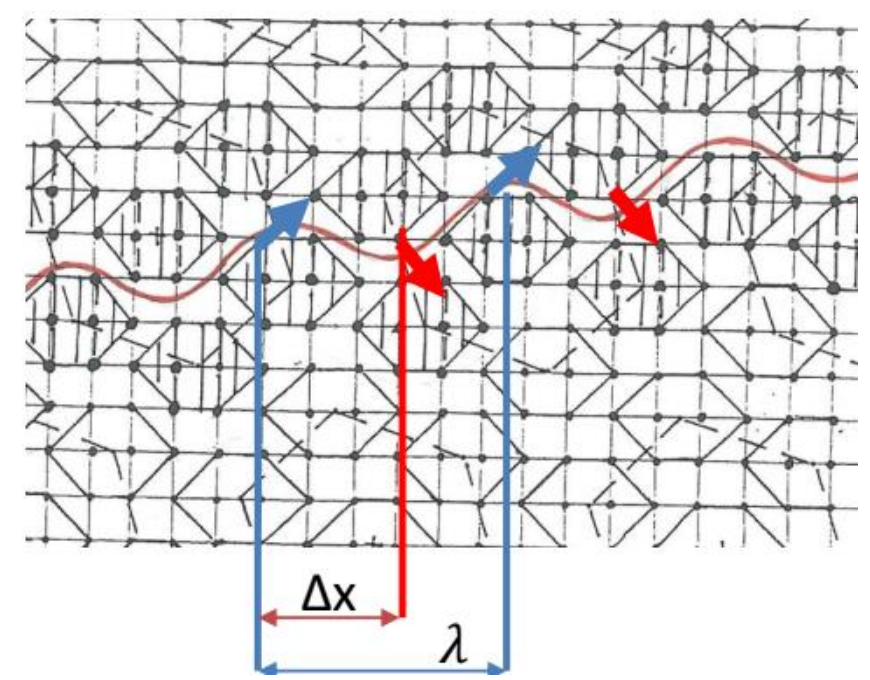

Fig.18 
In this model a photon is a particle to which space - USF gives "wave characteristics". When moving along the corridors of USF between its cells, the photon (and other particles) constantly interact with their "walls" - forms that make vibrations. When interacting with them, the photons will experience impacts that lead to both a straight forward movement along the corridor and a transverse movement from the "wall" to the "wall" (Fig.18). Interaction with the corridor particles is probably physically limited the maximum speed of movement of the smallest particle of matter -

The de Broglie hypothesis is the correctly guessed bandwidth of the USF corridors.

Fig. 19. Corridors of USF (2) with particles (1) with different "wavelength" $(\lambda)$, the distance from photon by the speed of light (c). In this case, the transverse displacements of the photon give it the appearance of a" transverse wave " with the polarization of oscillation $\mathrm{s}$ in a plane perpendicular to the direction of movement. For the same reason, other particles get wave properties also. With increasing the velocity $(\mathrm{V})$ or mass $(\mathrm{m})$ of the particle, the frequency of collisions increases, and the free path or wavelength $(\lambda)$ decreases accordingly, which explains the de Broglie hypothesis $\mathbf{m x V \mathbf { x }} \boldsymbol{\lambda}=\mathbf{h}$.

\section{Bandwidth of the space.}

impact to impact on the forms (3) that make up the walls of the corridor (2).

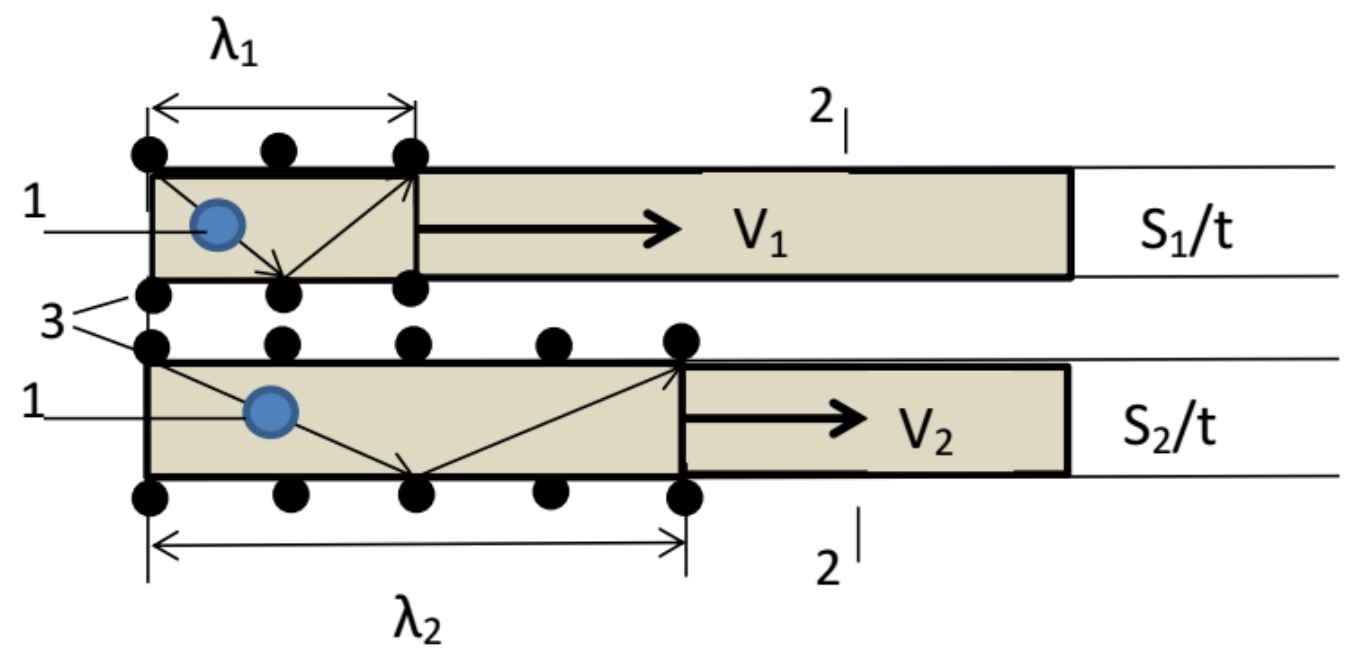

Fig. 20

Part of the length of the corridor $(\lambda)$, which the particle occupies from one impact against the wall of the corridor to the other, which cannot be occupied by another particle at the same time. Multiplied by the speed, it will be the area that will be occupied by the particle per unit of time $\lambda \cdot \mathrm{V}=\mathrm{S} / \mathrm{t}$.

This is how highway throughput is calculated for cars of different lengths moving at different speeds. Figure 19 shows the motion of two particles with different" wavelengths " $\lambda$ and velocities V. From the condition of limiting the throughput over the area $\mathbf{S}=\mathbf{S}_{\mathbf{1}}$ $=\mathbf{S}_{\mathbf{2}} ; \boldsymbol{\lambda}_{\mathbf{1}} \bullet \mathbf{V}_{\mathbf{1}}=\boldsymbol{\lambda}_{\mathbf{2}} \bullet \mathbf{V}_{\mathbf{2}}$. But here, on equal terms, another parameter arises that limiting the throughput - the mass of the particle $\mathbf{m x S} / \mathbf{t}=\mathbf{h}$.

The Heisenberg uncertainty principle is also determined by the capacity of the USF corridors. The particle moves along the corridor from impact on the image of one of its walls, to impact on the opposite wall at a half-wave distance $\Delta x=\lambda / 2$ at which its position is uncertain (fig. 18). From the de Broglie formula $\lambda=2 \cdot \Delta \mathbf{x}=\mathrm{h} / \mathrm{P}$ we obtain $\Delta \mathbf{x} \bullet \mathbf{p} \geq \mathbf{h} / \mathbf{2}$. As can be seen, the basic tenets of quantum mechanics find their clear explanation in the framework of the proposed model. Proceeding from it, the USF structures limit the movement of particles by the conditions $\dot{\boldsymbol{l}}=\mathbf{c}$ и $\mathbf{~} \mathbf{m} \cdot \dot{\boldsymbol{S}}=\mathbf{h}$.

\section{Inertia. Relativistic mass.}

The inertia phenomenon is also associated with the action of USF: with the displacement of any body, it meets the resistance of USF. At the initial moment, it is necessary not only to move the body, but also to deform this medium, rest inertia arises. At high speeds, the resistance of the University structures increases sharply. This may explain the increase in mass at nearlight speeds. Thus, the mass of the body can be represented as the sum of two components: the actual mass $\mathrm{m}$ and the resistance of the medium (USF) $\Omega$. Then $\mathrm{M}=\mathrm{m}+\Omega$, where $\Omega=\mathrm{m}\left(1 / \sqrt{ }\left(1-\mathrm{v}^{2} / \mathrm{c}^{2}\right)-1\right)$ [6].

When the body is already moving, the density of forms in the USF in front of it is higher than on the opposite side. And if you stop the action of an external force on a given body, which generated its movement, then it will continue to move due to the greater density of forms of the USF in front of the body, i.e., by inertia. 


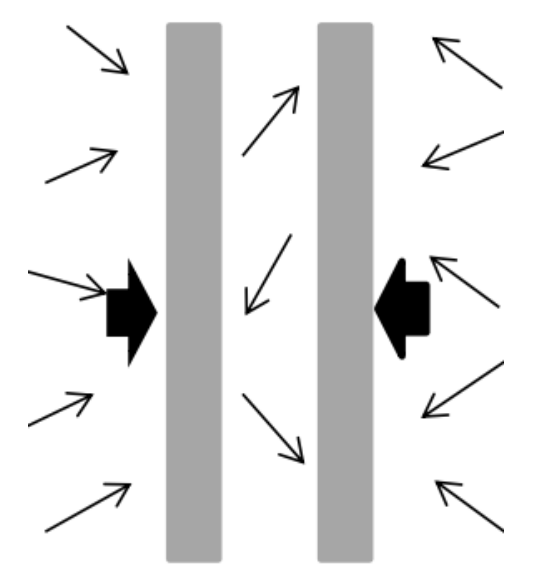

Fig.21.

\section{Structural vacuum.}

The closest to the concept of USF came the scientists involved in the structural vacuum. Modern physics unambiguously shows that vacuum is not a void, and a system of zero-point oscillations of quantum fields measured experimentally. This, in particular, is indicated by the Casimir effect, which consists in the mutual attraction of two parallel uncharged plates in a vacuum placed at a short distance. This is consistent with the proposed model of space, which is an oscillating structure of USF forms, which from free forms collect photons and other paired elementary particles striking the plates. Since there will be more such influences from the outside of the plates than from the inside, the plates will be attracted to each other (Fig.20).

\section{Stages and direction of development of the} world.

The creation of the Universe is a Unified process, at each new stage a new material structure or a new physical quality is formed. Starting with the combination of the two postulated primary particles forms, a qualitative and quantitative complication of matter occurs. Two types of particles are formed, one gets the possibility of independent existence, and the other a new physical quality and is involved in the further structuring of matter. Two forms, combining with parallel spins create an independent particle - a photon, and with antiparallel spins, a particle with a new quality em (mass element) (Fig.21). At the next level, it forms a particle with a real mass, and by another type, combined with a similar one in a rotational pair, it will create a particle with a new quality - a lepton number. This particle at the next level, combined with a particle of the previous level in mass and in a free form, will create an independent neutrino particle, and, by a different type, combined with a similar particle in a rotational pair, will create a particle with a new quality - electric charge (e/3).

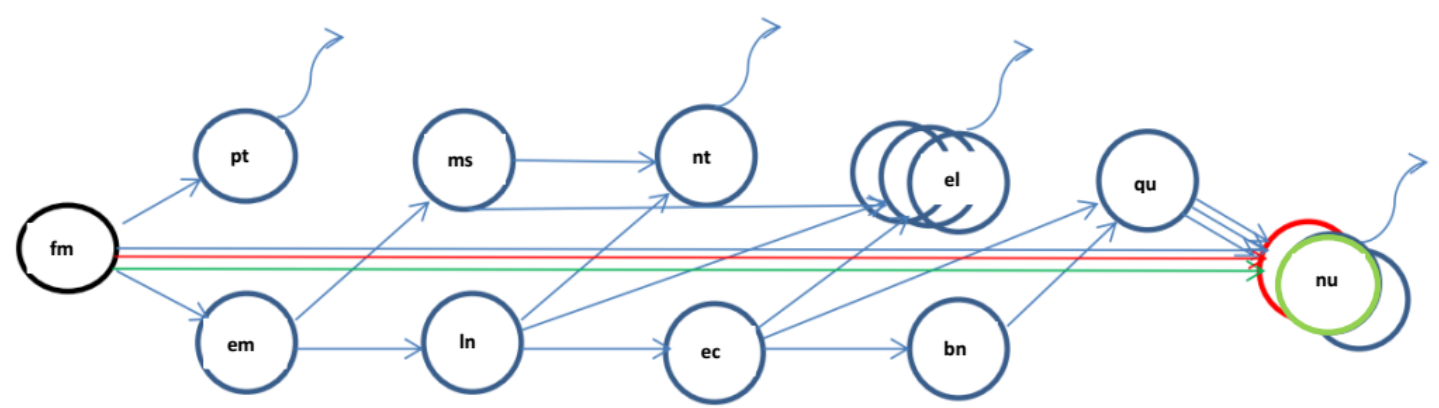

Fig.22. Diagram of the development of matter: fm-form; pt-photon; me-mass element; ms-mass; lp-lepton number; nt-neutrino; ec-electric charge; el-electron; bn-baryon number; qu-quark; ncnucleon.

At the next level, the union of three such particles, with the addition of particles of previous levels of mass and lepton number, form an independent electron particle. And according to another type, combining with a similar one with a new torque, it will create a particle with a baryon number (1/3). At the next level, this particle, combined with particles carrying an electric charge and mass, and attach a free form, will create a quark particle. An attached form can be one of three orthogonal orientations, which in accepted terminology gives them color charge. Further unification of three quarks with spins of different orientations (colors), according to the Pauli principle, 
will create nucleons (protons and neutrons). This completes the pre-atomic stage of structuring matter.

At the atomic stage, the complication of matter occurs due to the formation of new elements when electrons fill the corridors of the USF around the nuclei of atoms. The nuclei become heavier as long as they fit into the sizes of the primary cells of the USF. The next molecular stage is associated with the process of contraction of USF with the corresponding densification of atomic structures.

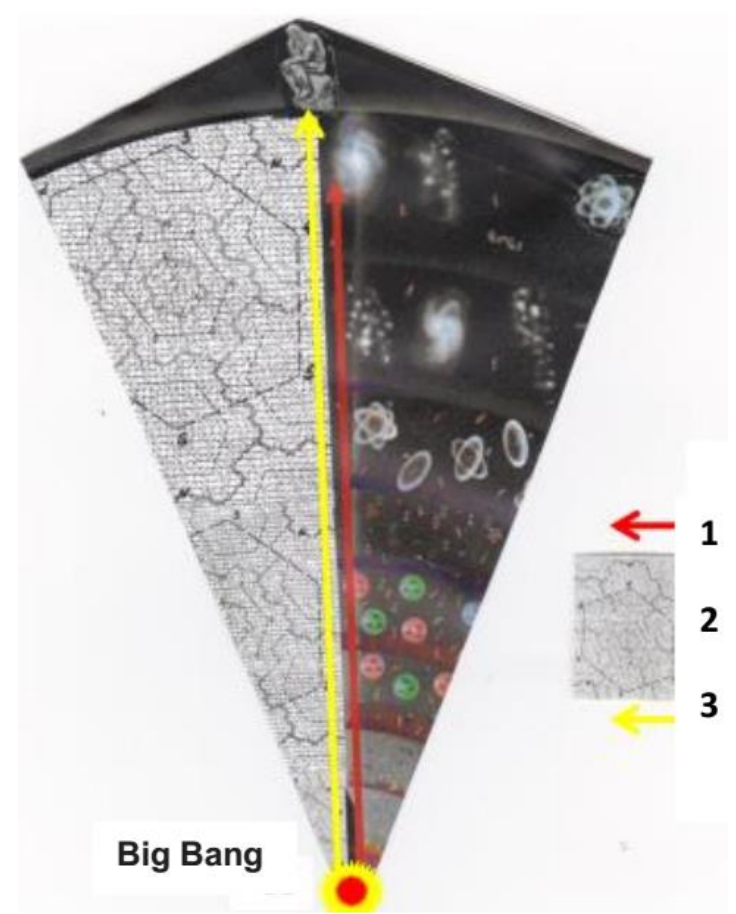

Fig.22. Universe evolution (Arrow of Time):

1.The development of structures of Unified set of forms (USF);

2. Structures of USF;

3. The development of material structures.

The collection and compaction of hydrogen molecules in clumps led to the formation, sequentially, of stars of the first, then second generation, in which heavy elements gradually formed. As a result of explosions of stars, they scattered in space. Of these, through a series of intermediate structures, the Solar system and the Earth formed, on which, in stages, the lithosphere, hydrosphere and atmosphere were formed. They created the conditions for the formation of organized matter at the next stage. All created material realities and interactions supplemented the rotation of the Earth, leading to the emergence in the USF of integral quasistationary cells that formed and scattered over time. They formed material structures that were also born and died. So a living cell arose, then a phased plant and animal world, mammals and finally man (Fig. 22). And all this complication of material structures was strictly controlled by USF structures, which ensured the coordination of the work of all structures of the Universe. In human society, the USF structure has become known as the spiritual half of the world.

This is already beyond the scope of physics. The authors understand that they will not be taken seriously by most scientists brought up on the traditions of quantum mechanics and theories of relativity. Despite this, we hope on the basis of the proposed model to create an open space for scientists of all specialties who are ready to take part in the creation of Unified Knowledge. Based on it, it is supposed to solve not only the physical problems of matter and space, but also to describe all world structures, including man, with his exclusive ability to cognize the world and determine its role in the Universe. The need for such knowledge is dictated by the current state of both world science and society.

\section{Bibliography:}

1. Prof. Einstein's adress at the University of Nottingham, Science, 71, 1930, 608-609

2. Unification of the four fundamental forces of nature by a binary quantum model. Reiner Georg Ziefle, Physics Essays, 29, 1 (2016)

3. Failure of the standard interpretation of quantum mechanics: Three experimental falsifications, and a consistent alternative interpretation. Reiner Georg Ziefle, Physics Essays, 30, 3 (2017)

4. The Big Bang or the Conductivity of the Interstellar Space Libor Neumann, Physical Science International Journal 23(1): 1-23, 2019; Article no.PSIJ.50051 ISSN: 2348-0130. DOI: 10.9734/PSIJ/2019/v23i130142

5. Romashov A. N. On the knowledge of the world. Moscow, 1998, $166 \mathrm{p}$.

6. Derivation of mass-energy equivalence using Newtonian mechanics as an alternative to Einstein's relativity postulates. Cyrus Master Khodabakhsha Physics Essays, 29,3 (2016) 